\title{
Posterior Pharyngeal Wall Schwannoma
}

\author{
${ }^{1}$ Varun Rai, ${ }^{2}$ Nikhil Arora, ${ }^{3}$ Vikas Malhotra, ${ }^{4} \mathrm{JC}$ Passey
}

\begin{abstract}
Case report of a posterior pharyngeal wall schwannoma in a 23-year-old female. Hypopharyngeal schwannoma is rare entities and at the time of writing we could find only 10 other cases reported worldwide and this is the eleventh. Complete excision was done intraorally and there is no recurrence after 1 year.
\end{abstract}

Keywords: Hypopharynx, Posterior pharyngeal wall, S 100 staining, Schwannoma.

How to cite this article: Rai V, Arora N, Malhotra V, Passey JC. Posterior Pharyngeal Wall Schwannoma. Int J Otorhinolaryngol Clin 2014;6(3):98-100.

Source of support: Nil

Conflict of interest: None

\section{INTRODUCTION}

A schwannoma is a benign encapsulated tumor arising from neural sheath cells called the Schwann cells. Approximately, 25 to $48 \%$ of these tumors are present in the head and neck with most arising from cranial nerves 9, 7, 11, 5 and 4 in that order. ${ }^{1}$ Schwannoma may be associated with neurofibromatosis and von Recklinghausen's disease. Schwannoma arising from posterior pharyngeal wall are extremely rare and present a diagnostic challenge to the otorhinolaryngologist. At the time of writing this paper, we could find only 10 other cases which histologically proven posterior pharyngeal wall schwannoma and this is the eleventh.

\section{CASE REPORT}

We present a case of a 28-year-old female who presented to the Outpatient Department (OPD) of Lok Nayak Hospital, New Delhi, India, with complaints of foreign body sensation in the throat since 3 months. She had no complaints of odynophagia, dysphagia or change in voice. She did not have any associated aural complaints.

Local examination of the oral cavity proved to be inconclusive and a videoendoscopic examination was

\footnotetext{
${ }^{1,2}$ Senior Resident, ${ }^{3,4}$ Professor

${ }^{1-4}$ Department of ENT, Lok Nayak Hospital, New Delhi, India
}

Corresponding Author: Varun Rai, Senior Resident, Department of ENT, H-465, New Rajinder Nagar, New Delhi 110060 India, Phone: +91-9958014000, e-mail: varun_rai@hotmail.com done in the OPD, which revealed a soft, pinkish, sessile, polypoidal mass arising from the posterior pharyngeal wall abutting the epiglottis (Fig. 1).

A contrast enhanced computed tomography (CECT) scan was ordered to rule out any malignant conditions ${ }^{2}$ or any vascular malformations in the area. The computed tomography (CT) scan revealed soft tissue attenuation of a polypoidal mass lesion projecting from posterior hypopharyngeal wall measuring $2.3 \times 1.4 \times 2.5 \mathrm{~cm}$ with minimal extension to right retropharyngeal wall causing mild attenuation of right pyriform sinus (Fig. 2).

The patient was worked up for surgery and was operated upon by the transoral route. Using Boyle Davis Mouth gag for visualization, the mass was excised in toto with sharp dissection and bipolar diathermy for hemostasis. The base of the mass was cauterized with

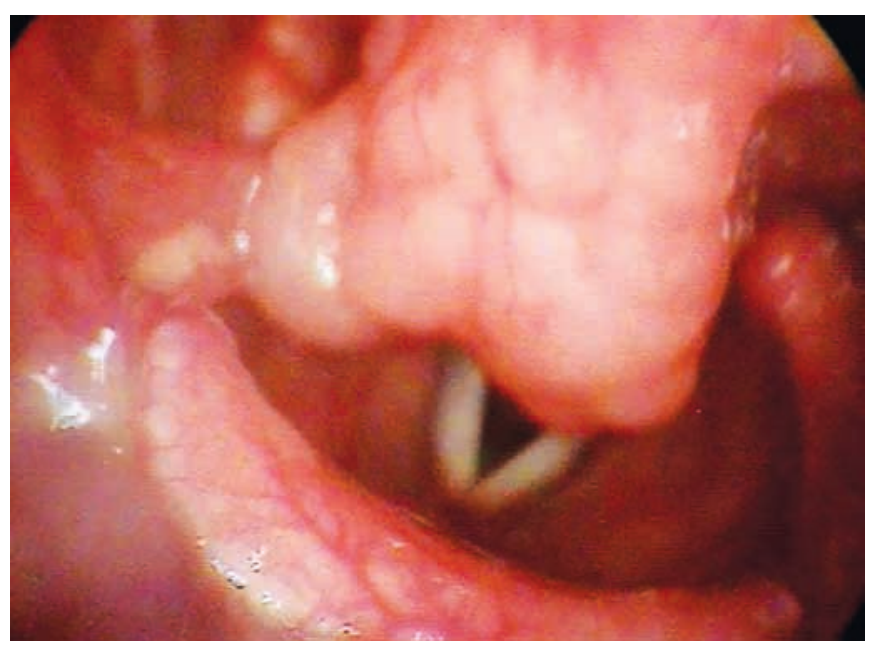

Fig. 1: Endoscopic view of the hypopharyngeal mass

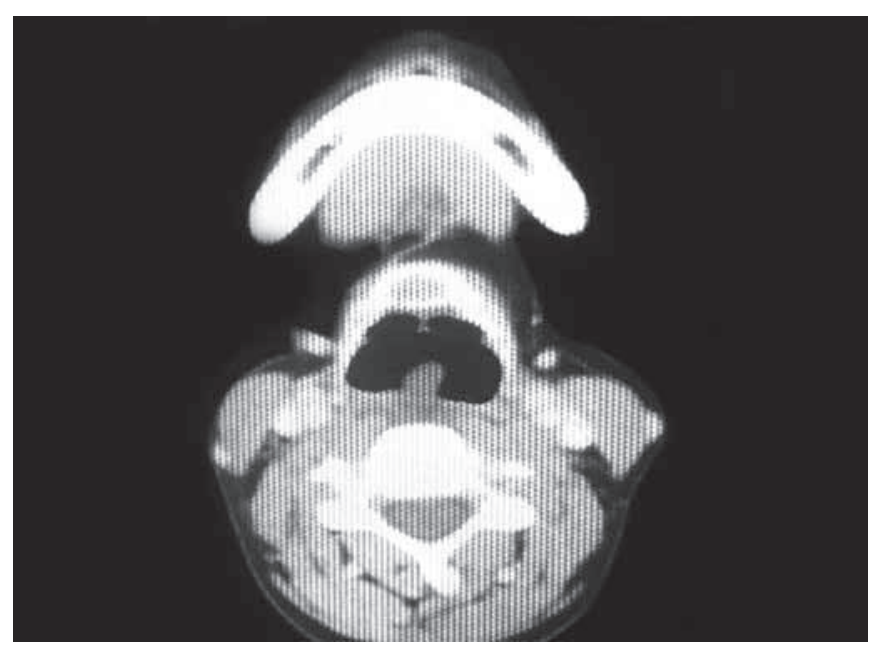

Fig. 2: Contrast-enhanced computed tomography scan image of the hypopharyngeal mass 
bipolar diathermy and we did not try to localize the nerve of origin. The patient was extubated uneventfully and put on Ryle's tube feeding for 3 days postoperative. She was started on an oral diet after 3 days, which she tolerated well. She was completely asymptomatic 3 weeks postsurgery and a repeat videoendoscopy was done which showed complete excision of tumor with complete healing and no residual tumor. She did not have any postoperative neural deficits.

Upon histologic examination irregular soft tissue of size $3 \times 2 \times 0.5 \mathrm{~cm}$ showed multiple nodules of tumor tissue comprising of predominantly hypercellular areas and occasional myxoid foci. Individual cells showed spindling and palisading, which stained intensely with S 100 staining along with characteristic Antoni A and B cells. A diagnosis of schwannoma was made upon the characteristic histopathologic finding and a positive S 100 stain (Fig. 3)

Due to financial constraints, a genetic karyotyping could not be done to rule out neurofibromatosis, however, clinically, the patient did not have any other lesions over the body or café au lait spots.

\section{DISCUSSION}

Schwannoma is slow growing benign encapsulated tumors, which grow along the cranial nerves. The pathogenesis involves proliferation of the Schwann cells lining the neural sheath. Although they can occur along any nerve, the most common cranial nerves affected according to occurrence are 9th, 7th, 11th, 5th and 4th The vagus, lingual, recurrent laryngeal nerves, posterior pharyngeal wall and the larynx are 'infrequently' affected sites.

Among the differentials of a polypoidal mass in the hypopharynx, hemangiomas, hemangioperiocytomas, neurofibromas, lipomas, fibromas and lymphangiomas must be considered. ${ }^{3}$

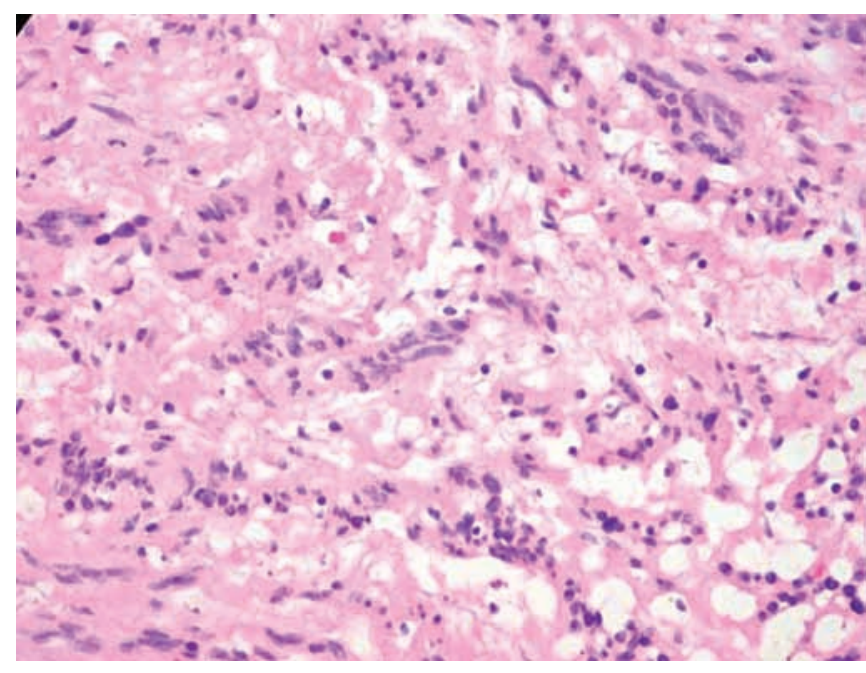

Fig. 3: Histopathologic picture of the schwannoma
The various symptoms associated with posterior pharyngeal wall schwannoma vary from foreign body sensation in throat, dysphagia, change in voice to respiratory difficulty. Slow progression of these symptoms point toward a more benign disorder.

The diagnostic tools in the surgeons armamentarium consist of a high index of suspicion as most of the symptoms are nonspecific and may be ignored by the patients and physicians alike, and visual confirmation which may be a simple indirect laryngoscopic examination or a videolaryngoscopic examination. We recommend a videolaryngoscopy where possible as this helps in accurate planning and recording the lesion. The only gold standard diagnostic investigation is histology of either biopsy or excised specimen.

Imaging, such as $\mathrm{CT}$ or magnetic resonance imaging (MRI) is invaluable in assessing the lesion. ${ }^{4}$ On CT, they appear isodense, but up to $20 \%$ of these tumors may display hypodense areas. On the other hand, with MRI, the lesion is characterized by intermediate intensity and can hardly be differentiated from muscle on T1 scans. On T2 scans, the intensity of a schwannoma increases. Therefore, the inhomogeneous structure and the cystic areas are represented by low intensity on T1 scans and as high intensity on T2 scans. Both of these imaging modalities also foretell extent of involvement of contiguous structures.

Surgical treatment is essentially the only option with preservation of the nerve function. ${ }^{5}$ Surgery involves complete excision or enucleation of the tumor. Various approaches have been employed, with the traditional open approach to the minimally invasive endoscopic transoral approach. We employed a transoral route with a mouth gag to facilitate surgical instrument access. However, in larger tumors not amenable to transoral approach a more traditional lateral pharyngotomy approach may be best. Tracing the nerve of origin has no effect on tumor clearance as long as the tumor does not encapsulate the nerve, as preservation of its function is paramount. ${ }^{6}$

Histologically, schwannoma has two different patterns: Antoni A and B areas. The former describes a cellular area in which sheets of spindle-shaped cells are often arranged in a palisading fashion, called Verocay bodies. The latter is composed of myxoid, loose, degenerative areas. Moreover, S 100 protein positivity with no demonstrable mitotic activity is suggested to be characteristic of these cellular areas. The histological pattern of this lesion with Antoni $A$ and $B$ as well as the $S 100$ protein expression helped to confirm the diagnosis of schwannoma in this patient. ${ }^{7}$

We are reporting this case due to its rarity and the diagnostic and therapeutic challenges, which are presented to the surgeon. 


\section{REFERENCES}

1. Koide C, Imai A, Takahashi T. Benign hypopharyngeal tumor: report of two cases. J Jpn Bronchoesophagol Soc 1992;43: 492-499.

2. Ecevit MC, Erdag TK, Guneri A, Sarioglu S, Guneri EA. A schwannoma mimicking hypopharyngeal carcinoma. Eur Arch Otorhinolaryngol 2006;263:469-472.

3. Caceres M, Steeb G, Wilks SM, Garrett HE Jr. Large pedunculated polyps originating in the esophagus and hypopharynx. Ann Thorac Surg 2006;81:393-396.
4. Pilavaki M, Chourmouzi D, Kiziridou A, Skordalaki A Zarampoukas T, Drevelengas A. Imaging of peripheral nerve sheath tumors with pathologic correlation: Pictorial review. Eur J Radiol 2004;52:229-239.

5. Ahmed AO, Umar AB, Aluko AA, Yaro MA. Hypopharyngeal schwannoma: a rare case presentation and review of literatures. Niger J Basic and Clin Sci 2013;10(1):29-32.

6. Ochiai A, et al. Hypopharyngeal neurinoma: report of two cases. Acta Otolaryngologica Suppl 2002;547:100-103.

7. Rosai J. Rosai and Ackerman's surgical pathology. 9th ed. Mosby-Elsevier: New York; 2004. 